COMUNICAÇÃO CIENTÍFICA

\title{
RENOVAÇÃO DO CARBONO-13 EM FIGUEIRAS 'ROXO DE VALINHOS'
}

\author{
ANDRÉA CARVALHO DA SILVA², ADILSON PACHECO DE SOUZA ${ }^{2}$ \\ CARLOS DUCATTI ${ }^{3}$, SARITA LEONEL ${ }^{4}$
}

RESUMO - O objetivo do trabalho foi determinar a taxa de renovação do carbono-13 ("turnover"), dos diferentes órgãos da figueira 'Roxo de Valinhos'. O experimento foi conduzido no pomar da Faculdade de Ciências Agronômicas, FCA/UNESP, Câmpus de Botucatu-SP. Determinou-se previamente, através das trocas gasosas com um medidor aberto portátil de fotossíntese, IRGA, a principal folha fotossinteticamente ativa. Essa folha foi colocada em uma câmara onde ocorreu a injeção do gás enriquecido. O tempo de enriquecimento da folha foi de 30 minutos. Os tratamentos foram constituídos por sete plantas de figueira, que foram retiradas do solo após: $6 ; 24 ; 48 ; 72 ; 120 ; 168$ e 360 horas do enriquecimento com ${ }^{13} \mathrm{C}$, e suas partes seccionadas em: gema apical, folha jovem, folhas adultas (fotossinteticamente ativas), brotações laterais, frutos e ramo. Os resultados obtidos permitiram o estabelecimento da sequência de metabolização do carbono-13 nas partições estudadas: Folhas novas $>$ Frutos $>$ Brotações $>$ Folhas Adultas $>$ Gema Apical $>$ Ramo $>$ Folha marcada. Plantas de figueira 'Roxo de Valinhos' apresentam reciclagem do ${ }^{13} \mathrm{C}$ de 24 horas e um tempo de meia-vida de duração do carbono-13 inferior a 11 horas.

Termos para indexação: Ficus carica, L., fisiologia, carboidratos, taxa de renovação.

\section{CARBON-13 TURNOVER IN 'ROXO DE VALINHOS' FIG TREES}

\begin{abstract}
The aim of this study was to assess carbon-13 turnover in different organs of the fig tree, 'Roxo de Valinhos' cultivar. The experiment was carried out in an orchard at School of Agronomical Sciences, FCA/UNESP, Botucatu Campus, State of São Paulo, Brazil. The main photosynthetically active leaf was previously determined based on gas exchanges by means of an open portable photosynthesis system, IRGA. That leaf was placed in a chamber where the enriched gas injection occurred. The leaf enrichment time was 30 minutes. Treatments were constituted of seven fig trees removed from the soil after: $6 ; 24 ; 48$; $72 ; 120 ; 168$ and 360 hours of enrichment using ${ }^{13} \mathrm{C}$, and their parts were sectioned into: apical bud, young leaves, adult leaves (photosynthetically active), lateral sprouts, fruits, and branch. The results allowed the establishment of the carbon-13 metabolism sequence in the studied parts: Young leaves $>$ Fruits $>$ Sprouts $>$ Adult leaves $>$ Apical bud $>$ branch $>$ Labeled leaf. 'Roxo de Valinhos' fig trees, had ${ }^{13} \mathrm{C}$ turnover of 24 hours and carbon-13 half-time shorter than 11 hours.
\end{abstract}

Index terms: Ficus carica L., phisiology, carbohydrates, turnover.

Os isótopos são elementos naturais que apresentam em sua constituição o mesmo número atômico, porém com números de massa diferentes, ou seja, apresentam o mesmo número de prótons e elétrons, e diferentes números de nêutrons, ocupando o mesmo lugar na tabela periódica. Dessa forma, os isótopos apresentam propriedades químicas semelhantes, sendo classificados em radioativos ou estáveis (DUCATTI et al., 2002).

De acordo com Ludlow et al. (1976), aproximadamente $99 \%$ de todo carbono na natureza está na forma do isótopo ${ }^{12} \mathrm{C}$ e apenas $1 \%$ estaria na forma do isótopo ${ }^{13} \mathrm{C}$. Estes dois isótopos estáveis do carbono comportam-se de forma diferente nas reações físicas e químicas, resultando em proporções variáveis destes isótopos nos diferentes materiais. Para Schimel (1995), os isótopos estáveis são usados para seguir movimentos e transformações químicas em sistemas biológicos e ambientais, podendo ser introduzidos na planta, no solo ou em sistemas aquáticos e monitorados com grande sensibilidade e precisão por espectrômetros de massa. Tornaramse uma ferramenta muito útil nas pesquisas sobre aspectos relacionados à fisiologia de plantas, uma vez

\footnotetext{
1(Trabalho 088-10). Recebido em: 31-03-2010. Aceito para publicação em: 12-08-2010.

${ }^{2}$ Prof. Adjunto, Instituto de Ciências Agrárias e Ambientais, UFMT, Campus de Sinop, Av. Alexandre Ferronatto 1200, Distrito Industrial, Cep: 78550-000, Sinop-MT. E-mails: acarvalho@ufmt.br, adilsonpacheco@ufmt.br

${ }^{3}$ Prof. Adjunto, Departamento Biofísica - IBB - UNESP, ducatti@ibb.unesp.br

${ }^{4}$ Prof. Adjunto, Departamento de Produção Vegetal, FCA/UNESP, Fazenda Lageado, Botucatu-SP, CEP: 18610-000, sarinel@fca.unesp.br
} 
que as razões entre estes dois isótopos podem auxiliar diretamente no estudo da fotossíntese, determinação dos ciclos fotossintéticos, translocação e alocação de carbono, e estresse hídrico, além de indiretamente servir de base no estudo sobre o melhoramento de plantas tolerantes ao estresse hídrico e mesmo para trabalhos relacionados ao desbaste e poda de plantas, notadamente de espécies frutíferas (EHLERINGER et al., 1993).

A determinação dos valores isotópicos, ou sinal isotópico nas diferentes espécies de plantas, é conseguido a partir de amostras do material orgânico da planta, que são analisadas pelo equipamento conhecido como espectrômetro de massas de razão isotópica (IRMS). Nesse sistema, de acordo com Ducatti et al. (2002), a amostra e o padrão são admitidos na forma de dióxido de carbono e, após a passagem por uma fonte de ionização, os feixes dos íons gerados são separados por um campo magnético de acordo com as suas relações massa/carga. Basicamente, compara-se a razão do ${ }^{13} \mathrm{CO}_{2}$ (massa 45) $/{ }^{12} \mathrm{CO}_{2}$ (massa 44) com uma amostra-padrão.

Atualmente, a informação das taxas de renovação do carbono-13 ("turnover") no metabolismo e nos órgãos de espécies vegetais são escassas, e os dados reportados restringem-se aos processos enzimáticos (COMPAROT-MOSS et al., 2010; DEWEZ et al., 2009; ALONSO et al., 2005; RUAN et al., 2004).

O ciclo fotossintético da figueira é do tipo C3 (TING et al., 1987; ZOTZ et al., 1997; CAN; AKSOY, 2007) e dependendo das condições ambientais, as plantas C3 apresentam atividade fotossintética variando entre 12 e $25 \mu \mathrm{mol} \mathrm{m} \mathrm{m}^{-2} \mathrm{~s}^{-1}$, corroborando Pigé et al. (2001) que, avaliando as características relacionadas à troca de $\mathrm{CO}_{2}$ em folhas e frutos de ramos da figueira com um ano de idade, durante a estação primavera-outono, observaram que a taxa fotossintética líquida variou de 15 a $20 \mu \mathrm{mol} \mathrm{m}^{-2} \mathrm{~s}^{-1}$. Visto que toda a produção de fitomassa e a formação da arquitetura da figueira dependem diretamente da atividade fotossintética, a prática da poda de frutificação é um manejo adotado nos pomares brasileiros, fazendo com a planta apresente uma renovação da parte aérea a cada ciclo produtivo (DALASTRA et al., 2009; SOUZA et al., 2009).

Nesse contexto, o objetivo deste trabalho foi determinar a taxa de renovação do carbono-13 ("turnover") dos diferentes órgãos da figueira cultivar Roxo de Valinhos.

O experimento foi conduzido no pomar da Faculdade de Ciências Agronômicas, FCA/UNESP, Câmpus de Botucatu-SP, latitude $22^{\circ} 51^{\prime} 55^{\prime \prime} \mathrm{S}$, longitude $48^{\circ} 27^{\prime} 22^{\prime \prime} \mathrm{O}$, a $810 \mathrm{~m}$ de altitude. O tipo climático predominante no local é o temperado quente (mesotérmico), com chuvas no verão e seca no inverno (CUNHA; MARTINS, 2009), com precipitação e temperatura média anual de $1.530 \mathrm{~mm}$ e $21^{\circ} \mathrm{C}$, respectivamente. O solo da área é classificado como Nitossolo Vermelho, segundo os critérios da Embrapa (1999). A área total do experimento é de $2.520 \mathrm{~m}^{2}$ e contém 190 plantas de figueira da cultivar Roxo de Valinhos, pomar implantado em setembro de 2001, com uma área de $6 \mathrm{~m}^{2} /$ planta, sendo estas conduzidas com 4 ramos.

Determinou-se previamente, através das trocas gasosas com um medidor aberto portátil de fotossíntese, IRGA, modelo LI-6400 (LI-COR), a principal folha fotossinteticamente ativa, sendo a quarta folha completamente expandida a contar do ápice do ramo, totalmente exposta à radiação solar, no período das $9 \mathrm{~h}$ às $11 \mathrm{~h}$ da manhã. Essa folha foi colocada em uma câmara de acrílico, tendo as seguintes dimensões: $28,0 \mathrm{~cm}$ de comprimento $\mathrm{x}$ $35 \mathrm{~cm}$ de largura $x 4,0 \mathrm{~cm}$ de altura $x 0,5 \mathrm{~cm}$ de espessura, perfazendo um volume de $4.000 \mathrm{~cm}^{3}$.

A injeção do gás enriquecido na câmara foi feita por meio de um dispositivo que regula $\mathrm{o}$ direcionamento do fluxo de um cilindro de ${ }^{13} \mathrm{CO}_{2}$ para uma seringa e, posteriormente, da seringa para a câmara, introduzindo-se $8,0 \mathrm{~mL}$ de ${ }^{13} \mathrm{CO}_{2}$, a 99 átomos \%, ou seja, de cada 100 átomos de $\mathrm{CO}_{2}$ deste gás, $99 \%$ dos átomos são formados por ${ }^{13} \mathrm{C}$ e $1 \%$ de ${ }^{12} \mathrm{C}$, que propiciaram um $\delta^{13} \mathrm{C}$ de $35.693,27 \%$ de ${ }^{13} \mathrm{C}$ (29,19 átomos $\%$ de ${ }^{13} \mathrm{C}$ em excesso). O tempo de enriquecimento da folha foi de 30 minutos, e após este período a câmara foi retirada.

Os tratamentos foram constituídos por sete plantas de figueira que foram retiradas do solo após: $6 ; 24 ; 48 ; 72 ; 120 ; 168$ e 360 horas do enriquecimento com ${ }^{13} \mathrm{C}$, e suas partes seccionadas em: gema apical, folhas jovens, folhas adultas (fotossinteticamente ativas), brotações laterais, frutos e ramo. Todas essas partes, assim que retiradas do solo, foram imersas em nitrogênio líquido $\left(-196^{\circ} \mathrm{C}\right)$ para provocar a morte celular.

No laboratório do Centro de Isótopos Estáveis Ambientais, IB/UNESP, Câmpus de Botucatu-SP, as amostras previamente identificadas foram colocadas em estufas com circulação de ar forçada a $65^{\circ} \mathrm{C}$, por 72 horas, para secagem. Posteriormente, na moagem, foi utilizado moinho criogênico à base de nitrogênio líquido (Spex - Modelo 6700). As amostras foram colocadas em tubos individualizados, imersos em nitrogênio líquido, para perfeita homogeneização. De cada amostra, foram retiradas $60 \mu \mathrm{g}$ e estas foram acondicionadas em cápsulas de estanho, com 06 $\mathrm{mm}$ de altura e $4 \mathrm{~mm}$ de diâmetro (Modelo D1106- 
Elemental Microanalysis Limited) e, em seguida, colocadas no analisador elemental (Carlo Erba EA 1108 - Fisons, Milão - Itália), acoplado ao espectrômetro de massas, para a realização da leitura do valor isotópico.

Das plantas em estádio reprodutivo, uma foi escolhida para a determinação do valor absoluto da caracterização natural $(-27,92 \%)$, para comparação com as plantas enriquecidas com ${ }^{13} \mathrm{C}$. As partições vegetativas da figueira enriquecida com carbono marcado apresentaram valores isotópicos distintos com o decorrer do tempo (0 a 360 horas após o enriquecimento). A alternância dos sítios de alocação entre elas, após determinado intervalo de tempo, gerou resultados de enriquecimento isotópico relativo, ou delta per mil ( $\delta \%$ ) versus tempo, com perfil aproximado de uma equação quadrática, específica para cada tecido (JONES et al., 1979). Era previsto que a continuidade dos valores isotópicos obtidos nas partições fosse descrita por uma função exponencial do tempo. Para Ducatti et al. (2002), a exploração desta função exponencial abre perspectivas de novas interpretações nestes experimentos, visando ao estudo da reciclagem de carbono-13 em tecidos vegetais.

Todos os fundamentos teóricos do desenvolvimento e aplicabilidade do modelo exponencial delta per mil $(\delta \%$ ) versus tempo foram apresentados por Ducatti et al. (2002), permitindo extrair apenas as equações utilizadas neste estudo, que relacionou a concentração inicial e a final de carbono-13 em uma partição com o tempo, conforme a equação 02 .

$$
\delta(\mathrm{t})=\delta_{\mathrm{FINAL}}+\left[\delta_{\text {INICIAL }}-\delta_{\mathrm{FINAL}}\right] \mathrm{e}^{(-\mathrm{Kt})}
$$

em que: $\delta_{\text {final }}$, representa o valor de delta per mil esperado após um tempo $\mathrm{t}$ (em horas); $\delta_{\text {inicial }}$, indica o valor de delta per mil proveniente da caracterização natural da partição. A constante $\mathrm{k}$ pode ser interpretada como a constante de troca isotópica com unidades de tempo ${ }^{-1}$.

Assim ocorrendo, à medida que o tempo aumenta, a concentração dos isótopos no tecido começa a ser substituída exponencialmente, ocorrendo a troca do carbono-13 (marcado) pelo carbono-12, dado pela discriminação natural das enzimas da planta pelo carbono-12.

$\mathrm{Na}$ condição de metade do enriquecimento final $(\delta \mathrm{f})$ e metade do enriquecimento inicial $(\delta \mathrm{i})$, pode-se obter, a partir da equação (2), o parâmetro de meia-vida $(\mathrm{T})$, expresso em unidade de tempo (horas) dado pela equação (3).

$$
T=\frac{\ln (2)}{K}
$$

A equação 3 pode ser será utilizada nas interpretações práticas da diluição isotópica nos diversos órgãos amostrados. Ducatti et al. (2002) expressam que, quanto menor a meia-vida $(\mathrm{T})$, maior será a taxa de renovação, ou o "turnover" do tecido. Por outro lado, admitindo que o valor de $\delta$ f seja maior do que o valor de $\delta$ i na equação 2 , pode-se expressar o tempo necessário para a troca de $95 \%$ dos átomos $(\mathrm{F})$, como exemplo, expresso pela equação 4 .

$$
t=-\frac{1}{K} \ln (1-F)
$$

Este parâmetro é empregado nas interpretações do percentual de troca $(\mathrm{F})$ do carbono nos diversos órgãos estudados da planta em questão (Tabela 1).

De forma geral, a constante $\mathrm{K}$ fornece uma ideia da "velocidade" do processo de eliminação ou troca do carbono-13 no tecido da partição. Como cada órgão da planta apresenta uma demanda e uma saída de fotoassimilados peculiares, essa constante foi diferente para cada partição estudada, assim como o valor de meia-vida também distinto para partições diferentes na mesma planta (Figura 1 e Tabela 2).

Os autores Manetta e Benedito-Cecilio (2003) relatam que o método de marcação isotópica, quando usado para a determinação da velocidade de processos metabólitos em animais, fornece resultados com respeito à taxa de "turnover". Um dos avanços mais significativos realizados com este método foi a descoberta de que os componentes macromoleculares das células e dos tecidos sofrem um processo constante de renovação metabólica. Existe um estado de equilíbrio dinâmico no interior celular, no qual a biossíntese é constante e exatamente contrabalançada por igual velocidade de degradação. Este processo de troca metabólica, "renovação celular", é também chamado de "lavagem celular", ou turnover.

A função exponencial descrita pela equação 02 apresentou ajuste excelente para predizer a relação delta per mil de carbono- $13 \delta^{13} \mathrm{C}$ versus tempo nas diversas partições vegetativas estudadas. $\mathrm{Na}$ Figura 1, podem ser observados os ajustes para a gema apical e frutos apenas do ramo que continha a folha enriquecida, permitindo inferir que a taxa de renovação de fotoassimilados, com o tempo, é maior para a gema apical, comparativamente ao órgão fruto, ou seja, a velocidade de troca dos fotoassimilados é maior na gema do que no fruto.

A expressão para a meia-vida sugere que partições que apresentam meia-vida maior, apresentam "baixa velocidade" de metabolização ou incorporação de carbono no tecido em questão, 
como o caso das folhas novas que apresentaram os maiores valores de $\delta{ }^{13} \mathrm{C}$ em todo o período estudado ( 0 a 360 horas). As folhas enriquecidas (folha 4) não atingiram valores negativos dentro das 360 horas estudadas; contudo, pela grande quantidade de carbono-13 assimilado pela folha e por ser a folha com maior atividade fotossintética da figueira, apresentaram os menores tempos de meia-vida. Os drenos não se diferenciaram quanto à taxa de turnover do carbono-13 (Tabela 1).

Por conseguinte, pode ser estabelecida a seguinte sequência de metabolização do carbono-13 nas partições estudadas: Folhas novas $>$ Frutos $>$ Brotações $>$ Folhas Adultas $>$ Gema Apical $>$ Ramo $>$ Folha marcada.
As equações ajustadas acima apresentaram bom desempenho na estimativa do carbono-13 trocado nas diferentes partições vegetativas estudadas no período de tempo entre 6 e 360 horas, conforme apresentado na Tabela 2. Evidenciou-se que praticamente em todas as partições, após 48 horas, acima de $95 \%$ do carbono já haviam sido trocados.

Entretanto, mesmo após 360 horas, os principais drenos, como gema apical, folhas novas e frutos apresentaram valores de $\delta^{13} \mathrm{C}$ que ainda caracterizavam o enriquecimento relativo, quando comparados com o valor absoluto da caracterização natural $(-27,92 \%$ ).

Plantas de figueira 'Roxo de Valinhos' apresentam reciclagem do ${ }^{13} \mathrm{C}$ de 24 horas e um tempo de meia-vida de duração do carbono-13 inferior a 11 horas.
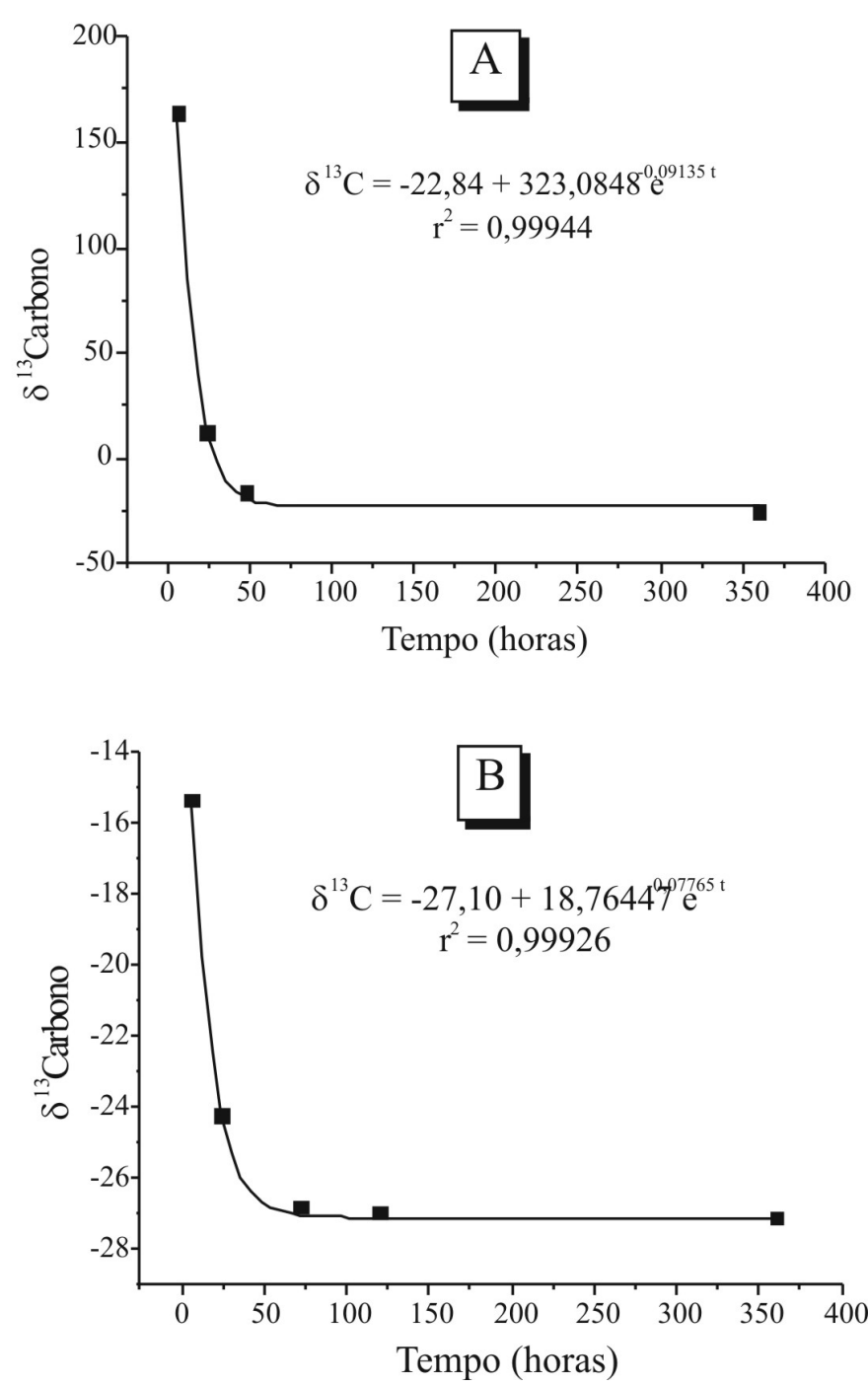

FIGURA 1 - Modelo exponencial para os isótopos estáveis do carbono em gema apical (a) e frutos (b) de Ficus carica L., considerando apenas o ramo da folha marcada. FCA / UNESP, Botucatu SP, 2008. 
TABELA 1 - Valores da equação exponencial experimental da taxa de renovação ("turnover") e da meiavida do carbono-13, em diferentes partições vegetativas de Ficus carica L. FCA/UNESP, Botucatu - SP, 2008.

\begin{tabular}{llcc}
\hline \multicolumn{1}{c}{ Partição } & \multicolumn{1}{c}{ Equação experimental ajustada } & $\mathrm{r}^{2}$ & $\begin{array}{c}\mathrm{t}_{1 / 2} \\
\text { (horas) }\end{array}$ \\
\hline Gema apical & $\delta{ }^{13} \mathrm{C}=-22,48+323,0484 \mathrm{e}^{-0,09135 t}$ & 0,99944 & 7,6 \\
Folhas Novas & $\delta{ }^{13} \mathrm{C}=-18,72+465,1661 \mathrm{e}^{-0,06267 t}$ & 0,99991 & 11,1 \\
Folha Marcada & $\delta{ }^{13} \mathrm{C}=5336,7219 \mathrm{e}^{-0,1422 \mathrm{t}}$ & 0,99986 & 4,9 \\
Folhas adultas & $\delta{ }^{13} \mathrm{C}=-28,94+7,5509 \mathrm{e}^{-0,0826 t}$ & 0,99991 & 8,4 \\
Brotação & $\delta{ }^{13} \mathrm{C}=-29,40+85,2482 \mathrm{e}^{-0,07924 t}$ & 0,99984 & 8,8 \\
Frutos & $\delta{ }^{13} \mathrm{C}=-27,10+18,7645 \mathrm{e}^{-0,07765 t}$ & 0,99926 & 8,9 \\
Ramo & $\delta{ }^{13} \mathrm{C}=-23,07+242,7526 \mathrm{e}^{-0,13532 t}$ & 0,99991 & 5,1 \\
\hline
\end{tabular}

TABELA 2 - Valores estimados e mensurados de $\delta \%{ }^{13} \mathrm{C}$ e a porcentagem de carbono- 13 trocado, para as diferentes partições vegetativas de Ficus carica L. FCA/UNESP, Botucatu-SP, 2008.

\begin{tabular}{|c|c|c|c|c|c|c|}
\hline \multirow[b]{2}{*}{ Tempo } & \multicolumn{3}{|c|}{ Gema apical } & \multicolumn{3}{|c|}{ Folhas jovens } \\
\hline & $\delta^{13} \mathrm{C}$ estimado & $\delta^{13} \mathrm{C}$ mensurado & $\begin{array}{l}\% \text { de } \mathrm{C} \\
\text { trocado }\end{array}$ & $\delta^{13} \mathrm{C}$ estimado & $\delta^{13} \mathrm{C}$ mensurado & $\begin{array}{l}\% \text { de C } \\
\text { trocado }\end{array}$ \\
\hline 6 & 163,92 & 164,29 & 42,2 & 300,60 & 300,65 & 31,4 \\
\hline$* \mathrm{~T}$ & 138,52 & - & 50,1 & 213,21 & - & 50,1 \\
\hline 24 & 13,23 & 12,45 & 88,8 & 84,58 & 84,64 & 77,8 \\
\hline 48 & $-18,81$ & $-16,37$ & 98,8 & 4,22 & $-13,78$ & 95,1 \\
\hline 72 & $-22,39$ & $-2,18$ & 99,9 & $-13,63$ & 24,25 & 98,9 \\
\hline 120 & $-22,83$ & 2,00 & 100 & $-18,47$ & $-8,64$ & 99,9 \\
\hline 168 & $-22,84$ & $-14,45$ & 100 & $-18,71$ & 7,55 & 100,00 \\
\hline 360 & $-22,84$ & $-25,07$ & 100 & $-18,72$ & $-18,72$ & 100,00 \\
\hline \multirow[b]{2}{*}{ Tempo } & \multicolumn{3}{|c|}{ Folha enriquecida } & \multicolumn{3}{|c|}{ Folhas adultas } \\
\hline & $\delta^{13} \mathrm{C}$ estimado & $\delta^{13} \mathrm{C}$ mensurado & $\begin{array}{c}\% \text { de } \mathrm{C} \\
\text { trocado }\end{array}$ & $\delta^{13} \mathrm{C}$ estimado & $\delta^{13} \mathrm{C}$ mensurado & $\begin{array}{l}\% \text { de C } \\
\text { trocado }\end{array}$ \\
\hline 6 & 2273,71 & 2273,97 & 57,4 & $-24,34$ & $-24,53$ & 39,1 \\
\hline$* \mathrm{~T}$ & 2658,68 & - & 50,2 & $-25,17$ & - & 50,0 \\
\hline 24 & 175,84 & 175,92 & 96,7 & $-27,90$ & $-27,13$ & 86,2 \\
\hline 48 & 5,79 & 33,02 & 99,9 & $-28,80$ & $-29,36$ & 98,1 \\
\hline 72 & 0,19 & 5,01 & 100,00 & $-28,92$ & $-29,17$ & 99,7 \\
\hline 120 & 0,00 & 15,05 & 100,00 & $-28,94$ & $-29,17$ & 100,00 \\
\hline 168 & 0,00 & 18,05 & 100,00 & $-28,94$ & $-29,32$ & 100,00 \\
\hline 360 & 0,00 & 21,05 & 100,00 & $-28,94$ & $-28,96$ & 100,00 \\
\hline \multirow[b]{2}{*}{ Tempo } & \multicolumn{3}{|c|}{ Frutos } & \multicolumn{3}{|c|}{ Brotações } \\
\hline & $\delta^{13} \mathrm{C}$ estimado & $\delta^{13} \mathrm{C}$ mensurado & $\begin{array}{l}\% \text { de } \mathrm{C} \\
\text { trocado }\end{array}$ & $\delta^{13} \mathrm{C}$ estimado & $\delta^{13} \mathrm{C}$ mensurado & $\begin{array}{l}\% \text { de } \mathrm{C} \\
\text { trocado }\end{array}$ \\
\hline 6 & $-15,32$ & $-15,32$ & 37,2 & 23,59 & 23,59 & 37,8 \\
\hline$* \mathrm{~T}$ & $-17,70$ & - & 49,9 & 13,05 & - & 50,2 \\
\hline 24 & $-24,19$ & $-24,21$ & 84,5 & $-16,67$ & $-29,5$ & 85,1 \\
\hline 48 & $-26,65$ & $-27,54$ & 97,6 & $-27,50$ & $-27,5$ & 97,8 \\
\hline 72 & $-27,03$ & $-26,8$ & 99,6 & $-29,12$ & $-29,59$ & 99,7 \\
\hline 120 & $-27,10$ & $-26,95$ & 99,9 & $-29,39$ & $-18,76$ & 99,9 \\
\hline 168 & $-27,10$ & $-27,78$ & 100,00 & $-29,40$ & $-29,4$ & 100,00 \\
\hline 360 & $-27,10$ & $-27,1$ & 100,00 & $-29,40$ & $-28,83$ & 100,00 \\
\hline
\end{tabular}

*(T) tempo de meia-vida da respectiva partição vegetativa. 


\section{REFERÊNCIAS}

ALONSO, A. P.; VIGEOLAS, H.; RAYMOND, P.; ROLIN, D.; DIEUAIDE-NOUBHANI, M.A New Substrate Cycle in Plants. Evidence for a High Glucose-Phosphate-to-Glucose Turnover from in Vivo Steady-State and Pulse-Labeling Experiments with $\left[{ }^{13} \mathrm{C}\right]$ Glucose and $\left[{ }^{14} \mathrm{C}\right]$ Glucose. Plant Physiology, Minneapolis, v.138, p. 2220-2232, 2005.

CAN, H. Z.; AKSOY, U. Seasonal and diurnal photosynthetic behaviour of fig (Ficus carica L.) under semi-arid climatic conditions. Acta Agriculturae Scandinavica, Stockholm, v.57, n.4, p.297-306, 2007.

COMPAROT-MOSS, S.; KOTTING, O.; STETTLER, M.; EDNER, C.; GRAF, A.; WEISE, S. E.; STREB, S.; LUE, W. L.; MACLEAN D.; MAHLOW, S.; RITTE, G.; STEUP, M.; CHEN, J.; ZEEMAN, S.C.; SMITH, A. M. A Putative Phosphatase, LSF1, Is Required for Normal Starch Turnover in Arabidopsis Leaves. Plant Physiology, Minneapolis, v.152, p. $685-697,2010$.

CUNHA, A. R.; MARTINS, D. Classificação climática para os municípios de Botucatu e São Manuel. Irriga, Botucatu, v.41, p.1-11, 2009.

DALASTRA, I. M.; PIO, R.; CAMPAGNOLO, L. A.; DALASTRA. G.A.; CHAGAS, E. A.; GUIMARÃES, V.F. Épocas de poda na produção de figos verdes 'Roxo de Valinhos' em sistema orgânico na região oeste do Paraná. Revista Brasileira de Fruticultura, Jaboticabal, v.31, n.2, p.447-453, 2009.

DEWEZ, D.; PARK, S.; CERDÁN, J. G. G.; LINDBERG, P.; MELIS, A. Mechanism of REP27 Protein Action in the D1 Protein Turnover and Photosystem II Repair from Photodamage. Plant Physiology, Minneapolis, v. 151, p. 88-99, 2009.

DUCATTI, C.; CARRIJO, A. S.; PEZZATO, A. C.; MANCERA, P. F. A. Modelo teórico e experimental da reciclagem do carbono-13 em tecidos de mamíferos e aves. Scientia Agricola, Piracicaba, v. 59, n. 1, p. 29-33, jan./mar. 2002.

EHLERINGER, J. R.; HALL, A. E.; FARQUHAR, G. D. Stable isotopes and plant carbon-water relations. New York: Academic Press, 1993. 555 p.

EMBRAPA. Centro Nacional de Pesquisa de Solos. Sistema brasileiro de classificação de solos. Rio de Janeiro, 1999. 412 p.
JONES, R. J.; LUDLOW, M. M.; TROUGHTON, J. H.; BLUNT, C. G. Estimation of the proportion of C3 and $\mathrm{C} 4$ plant species in the diet of animals from the ratio of natural $12 \mathrm{C}$ and $13 \mathrm{C}$ isotopes in the faeces. Journal of Agricultural Science, Cambridge, v.92, p.91-100, 1979.

LUDLOW, M. M.; TROUGHTON, J. H.; JONES, R. $\mathrm{J}$. Atechnique for determining the proportion of $\mathrm{C} 3$ and $\mathrm{C} 4$ species in plant samples using stable natural isotopes of carbon. Journal Agriculture Science, Cambridge, v. 87, p. 625-632, 1976.

MANETTA, G. I.; BENEDITO-CECILIO, E. Aplicação da técnica de isótopos estáveis na estimativa da taxa de turnover em estudos ecológicos: uma síntese. Acta Scientiarum: Biological Sciences, Maringá, v. 25, n. 1, p. 121-129, 2003.

PIGÉ, L.G.; SALAGER, J. L.; MCKEY, M. H.; ROY, J. Carbon allocation to volatiles and other reproductive componentsin male Ficus carica (Moraceae). American Journal of Botany, Columbus, v.88, p.2214-2220, 2001.

RUAN, Y. L.; XU, S. M.; WHITE, R.; FURBANK, R. T. Genotypic and Developmental Evidence for the Role of Plasmodesmatal Regulation in Cotton Fiber Elongation Mediated by Callose Turnover. Plant Physiology, Minneapolis, v.136, p.4104-4113, 2004.

SCHIMEL, D.S. Terrestrial ecosystems and the carbon cycle. Global Change Biology, Oxford, v. 1, p. 77-91, 1995.

SOUZA, A. P.; SILVA, A. C.; LEONEL, S.; ESCOBEDO, J. F. Temperaturas basais e soma térmica para a figueira podada em diferentes épocas. Revista Brasileira de Fruticultura, Jaboticabal, v.31, n.2, p.314-322, 2009.

TING, I. P.; HANN, J.; HOLBROOK, N. M.; PUTZ, F. E.; STERNBERG, L. da S.L.; PRICE, D.; GOLDSTEIN, G. Photosynthesis in hemiephiphytic species of Clusia and Ficus. Oecologia, Berlin, v.74, p.339-346, 1987.

ZOTZ, G.; PATINÕ, S.; TYREE, M. T. Water relations and hydraulic architecture of woody hemiepiphytes. Journal of Experimental Botany, Oxford, v.48, n.10, p.1825-1833, 1997. 